\title{
Valoración de la eficacia de las mallas libres de tensión y satisfacción de 241 pacientes en la corrección de la incontinencia urinaria
}

\author{
Zapardiel Gutiérrez I, De la Fuente Valero J, Botija Botija J, Pérez Medina T, Díez Fernández M, \\ Bajo Arenas JM.
}

\author{
Departamento Obstetricia y Ginecología. Hospital Universitario Santa Cristina. Madrid
}

Actas Urol Esp. 2008;32(6):637-641

\section{RESUMEN}

VALORACION DE LA EFICACIA DE LAS MALLAS LIBRES DE TENSIÓN Y SATISFACCION DE 241 PACIENTES EN LA CORRECCION DE LA INCONTINENCIA URINARIA

Introducción: La incontinencia urinaria es un problema muy extendido entre mujeres de mediana edad, para su corrección se han descrito muchas técnicas en los últimos años con diferentes tasas de éxito y complicaciones. Nuestro objetivo es valorar la eficacia de la mallas libres de tensión transobturadoras (TOT) en la corrección de este problema junto con la satisfacción de las pacientes con el procedimiento.

Material y métodos: Estudio retrospectivo en 241 mujeres a las que se las corrigió su incontinencia urinaria con malla TOT. Se analizó la tasa de éxito y la tasa de satisfacción de las pacientes a los 2 y 6 meses tras la cirugía. Además se recogieron las tasas de complicaciones a corto y largo plazo.

Resultados: La tasa de continencia de las pacientes a los 2 meses estuvo en torno el $70 \%$ y la de satisfacción en torno al 65\%. A los 6 meses fueron del 70\% y 70\% respectivamente, variando ligeramente según el procedimiento asociado. Las tasa de complicaciones intraoperatorias fue del 3,31\% y postoperatorias del $26,55 \%$ siendo casi todas ellas complicaciones menores.

Discusión: La TOT es una opción eficaz y útil en la corrección de la incontinencia urinaria con una baja tasa de complicaciones. Además con una alta tasa de satisfacción de las pacientes.

Palabras clave: Incontinencia Urinaria. TVT. Banda transobturadora.

\section{ABSTRACT \\ EFFECTIVENESS EVALUATION OF TENSION-FREE VAGINAL TAPES IN THE TREATMENT OF URINARY INCONTINENCE AND SATISFACTION OF 241 PATIENTS}

Introduction: Urinary incontinence is an extended problem between middle-aged women. There are several options for its correction with different effectiveness and complication rates. Our objective is to evaluate the effectiveness of tension-free vaginal transobturator tapes (TOT) in the repair of this incontinence. Also to evaluate the patients satisfaction rate with this kind of surgery.

Material and Methods: Retrospective study in 241 women with a surgical repair of their urinary incontinence with TOT. We analyzed the rates of effectiveness and satisfaction of the patients with the surgical repair after 2 and 6 months. The complication rate was also analyzed.

Results: Urinary continence rate 2 months after surgery was around $70 \%$ and the satisfaction one around $65 \%$. After 6 months the rates were $70 \%$ and $70 \%$ respectively with a minimal variation depending on the associated surgery. The complication rate during surgery was $3,31 \%$ and the one after surgery was $26,55 \%$, although most of them were minor complications.

Comment: The use of TOT for the repair of urinary incontinence is an effective and safe option with a low complication rate. Moreover the rate of patients satisfaction is high.

Keywords: Urinary incontinence. Tensión Free Vaginal Tape. Transobturator tape.

$\mathrm{E}$ la literatura médica hay descritas una gran variedad de técnicas quirúrgicas para la incontinencia urinaria de las mujeres. Las primeras técnicas mínimamente invasivas para la corrección quirúrgica de la incontinencia urinaria de esfuerzo datan de la década de 1950. Desde entonces hasta ahora ha habido numerosas innovaciones encaminadas a obtener los resultados conseguidos con la cirugía abierta pero con una menor morbilidad. De todas estas técnicas los cabestrillos ("slings") suburetrales 
libres de tensión han sido los más utilizados, especialmente la clásica TVT que se ha convertido en la opción más empleada para la corrección quirúrgica de la incontinencia urinaria de esfuerzo en el mundo entero. Las técnicas de slings han sufrido varias modificaciones, que básicamente se pueden agrupar en cuatro categorías: composición del cabestrillo, posición del cabestrillo (cuello vesical o sub-uretra media), técnica de colocación y grado de tensión del cabestrillo. En 1995, Ulmsten U y Petros $\mathrm{P}^{1}$ revolucionan el planteamiento terapéutico con una técnica quirúrgica mínimamente invasiva (TVT: tensión free vaginal tape), eficaz (tasas de curación en torno al 85-90\%) pero no exenta de complicaciones intraoperatorias importantes (lesiones vesicouretrales e intestinales) que ensombrecen sus resultados. En el año 2001, Delorme E. et $\mathrm{al}^{2}$ presenta una modificación de este procedimiento en un intento de evitar la perforación accidental de la vejiga, y por tanto, la necesidad de realizar una exploración endoscópica intraoperatoria; hablamos de la TVT transobturador o TOT. Las agujas se van a pasar desde la cara interna del muslo hasta la región vaginal parauretral a través del agujero obturador y los músculos puborrectales. Se han descrito dos abordajes diferentes: desde el muslo hasta la vagina (técnica “out-in”) y en dirección contraria (“in-out") 3 .

Nuestro objetivo es evaluar la eficacia de la malla TOT en la corrección de la incontinencia urinaria (IU), asociada o no a prolapso urogenital. Como objetivos secundarios nos planteamos el conocer la seguridad del proceso, así como la aceptabilidad y satisfacción por parte de la paciente.

\section{MATERIAL Y MÉTODOS}

Se incluyen en este estudio retrospectivo, 241 mujeres, que desde Enero de 2004 a Enero de 2007, fueron sometidas a tratamiento quirúrgico de su incontinencia urinaria mediante un dispositivo TOT, asociándose la práctica de diversas técnicas indicadas en el prolapso urogenital, si la situación clínica lo aconsejaba.

La edad media de las pacientes fue de 59,26 $\pm 11,02$ años (34-86). La multiparidad ( $\geq 2$ partos vaginales) fue una característica presente en el 90,04\% (217) de las mujeres. Un 83,40\% (201) eran menopáusicas. Las características de las pacientes en cada uno de los grupos de incontinencia urinaria se detallan en la Tabla 1.

Cada una de las pacientes fue evaluada por un tándem ginecólogo-urólogo; tras una minuciosa anamnesis, exploración física y diversas pruebas complementarias (ecografía genitourinaria, estudio sanguíneo completo y analítica de orina), es el estudio urodinámico (EUD) quien establece las siguientes categorias de incontinencia urinaria (asociadas o no a diversos tipos y grados de prolapso urogenital): incontinencia urinaria de esfuerzo (IUE), incontinencia urinaria de urgencia (IUU), incontinencia urinaria mixta (IUM), no IU. Este último grupo corresponde a mujeres con clínica de IU pero con un EUD normal. Los casos de vejiga hiperactiva fueron tratados con mediación anticolinérgica durante varias semanas antes de la cirugía.

Las opciones de tratamiento que se establecieron fueron cuatro: TOT aislada $(n=94)$, TOT + corrección de prolapso (malla intravaginal o colporrafia) $(n=88)$, TOT más histerectomía vaginal $(\mathrm{HV})(\mathrm{n}=12)$ y TOT + corrección de prolapso más histerectomía vaginal $(\mathrm{n}=47)$.

Todos los procedimientos se realizaron en quirófano, bajo anestesia general o raquídea; el equipo quirúrgico estuvo formado por ginecólogos, urólogos y en ocasiones residentes de ginecología.

El dispositivo TOT empleado fue el comercializado por Ethicon: Ginecare TVT Obturador System (Ethicon, Inc) (Fig. 1). Este Kit consta de los siguientes elementos:

Tabla 1. Edad media y multiparidad en función del tipo de incontinencia urinaria

\begin{tabular}{lccc}
\hline & n (\%) & $\begin{array}{c}\text { Edad media } \pm \\
\text { desviación estándar } \\
\text { (rango) }\end{array}$ & $\begin{array}{c}\text { Multiparidad } \\
\mathbf{n}(\%)\end{array}$ \\
\hline IUE & $134(55,60)$ & $58,39 \pm 11,31(35-86)$ & $118(88,05)$ \\
IUU & $5(2,07)$ & $68 \pm 3,67(64-74)$ & $3(60,00)$ \\
IUM & $48(19,91)$ & $58,15 \pm 10,18(34-79)$ & $45(93,75)$ \\
No IU & $54(22,40)$ & $61,57 \pm 10,96(38-85)$ & $51(94,44)$ \\
\hline
\end{tabular}

- Banda de polipropileno de 1,1x45 cm con una vaina de plástico superpuesta en el medio y receptáculos tubulares plásticos en cada extremo.

- Dos pasadores helicoidales de alambre, curvos, de acero inoxidable con mangos de plástico, previamente montados a la banda de polipropileno. 


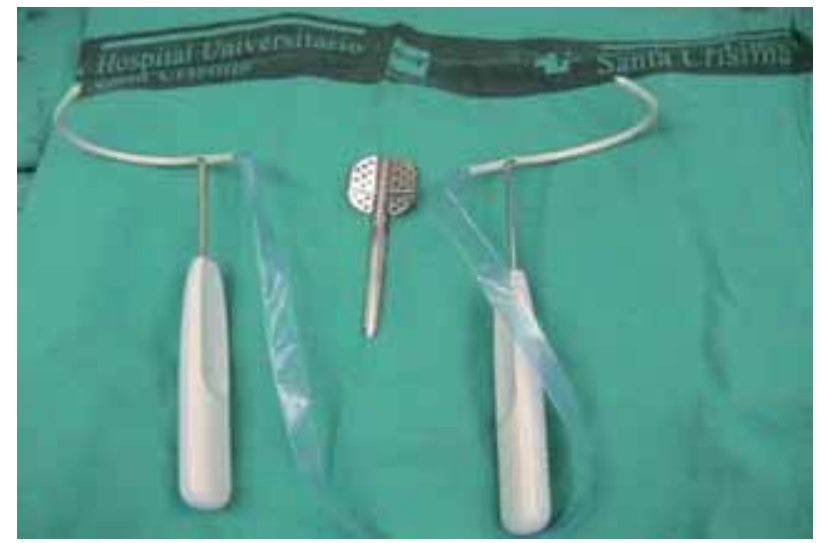

FIGURA 1. Dispositivo Ginecare TVT Obturador System.

- Una guía con aletas atraumáticas, de acero inoxidable, que facilita el pasaje de los pasadores helicoidales por el área de disección.

Las mallas vaginales empleadas en los casos necesarios también pertenecían a la misma casa comercial.

La aplicación del dispositivo TOT se realizó según los principios de la técnica descrita por Delorme en el año 2001 pero con un sentido IN-OUT (Fig. 2). La histerectomía vaginal y las diferentes colporrafias se realizaron de acuerdo a las técnicas descritas al uso. No fue necesario realizar ninguna cistoscopia intraoperatoria.

Durante la estancia hospitalaria se realizaron controles analíticos hematológicos y urinarios. Las pacientes fueron evaluadas en la consulta de urología del hospital al mes, dos y seis meses desde el procedimiento. La valoración de la continencia y satisfacción postquirúrgicas se determinó a través de entrevista personal, en la consulta, con cada una de las pacientes.

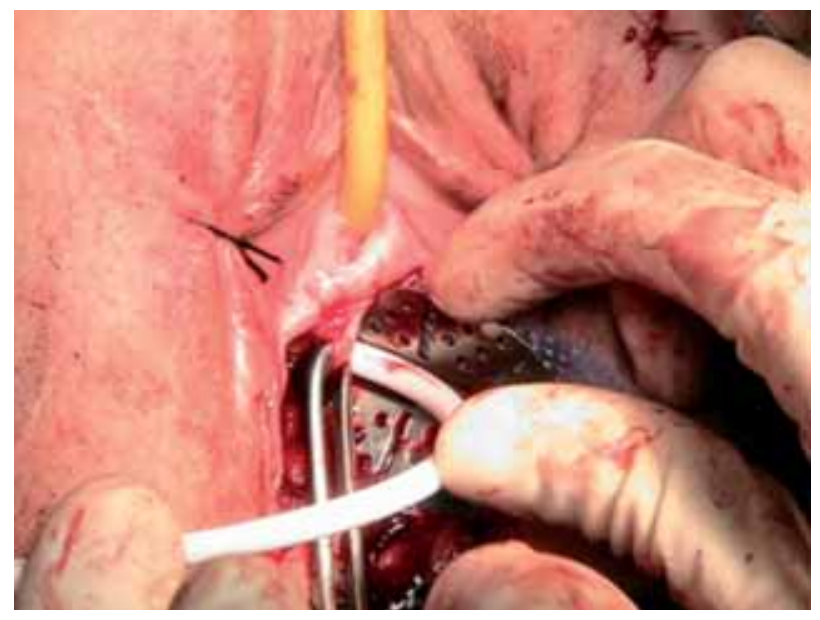

FIGURA 2. Aplicación de TOT en dirección IN-OU.

\section{RESULTADOS}

La tasa global de perforación vesical fue de 2,48\% (6 mujeres); el diagnóstico de esta complicación fue intraoperatorio al evidenciar salida de líquido vesical tras aplicar los pasadores. Todas ellas se solventaron con puntos sueltos vesicales intraoperatoriamente y sondaje vesical permanente durante 7 días más cobertura antibiótica. 12 mujeres (4,97\%) sufrieron extrusión vaginal de la malla de TOT, siendo reparadas quirúrgicamente 11 de ellas con anestesia locorregional, extirpando el fragmento extrusionado hacia vagina, refrescando los bordes quirúrgicos y cerrando la pared vaginal. La última se recortó de manera ambulatoria ya que el fragmento extrusionado era mínimo y bastó con anestesia local. Las tasas del resto de complicaciones se detallan en la Tabla 2. Cabe resaltar que la laceración uretral se produjo accidentalmente durante la disección de la zona, pero no necesitó sutura solventándose con sondaje 15 días. Tanto las extrusiones, como las perforaciones como la laceración cursaron posteriormente con normalidad sin secuelas en las subsiguientes revisiones. Con respecto al cuadro tetánico fue de causa infecciosa que aparición a los 10 días de la cirugía y se solventó posteriormente con antibioterapia y benzodiacepinas sin incidencias. Con respecto a los prolapsos, uno fue de cúpula tras histerectomía vaginal y el resto fueron uterinos que se reevaluaron y pasaron a protocolo de prolapso uterino sin establecerse una relación causal clara con la cirugía de incontinencia. Los dolores en el área inguinal y quirúrgica suponen casi el 10\% de las complicaciones, siendo estos leves y moderados difusos que se solventan en su mayor parte con analgésicos orales. Las parestesias de MMII (2 casos) que surgieron duraron ambas alrededor de 2 días y se relacionaron con la postura forzada en quirófano, ambas se solucionaron espontánea y gradualmente en varios días. Las 4 retenciones urinarias surgidas se resolvieron en 5 y 6 días durante los cuales se realizaron vaciamientos repetidos con sonda desechable. Los hematomas fueron superficiales y también se resolvieron espontáneamente y hobo 4 infecciones de la herida quirúrgica que cedieron con antibioterapia de amplio espectro, al igual que la infección urinaria surgida. Por último en relación a los sangrados posquirúrgicos, fueron de escasa cuantía y el que no cedió espontáneamente se solventó con taponamiento vaginal 48 horas. 
Tabla 2. Complicaciones intra y postoperatorias de las diferentes técnicas

\begin{tabular}{|c|c|c|c|c|c|}
\hline & TOT & TOT $+\mathrm{CP}$ & TOT $+\mathrm{HV}$ & TOT+HV+CP & \% Global \\
\hline Intraoperatorias & & & & & 3,31 \\
\hline Perforación vesical & 4 & 2 & 0 & 0 & 2,48 \\
\hline Laceración uretral & 0 & 0 & 1 & 1 & 0,82 \\
\hline Posoperatorias & & & & & 26,55 \\
\hline Dolor & 7 & 9 & 2 & 6 & 9,95 \\
\hline Hematoma punción & 3 & 5 & 1 & & 3,73 \\
\hline ITU & 1 & & & & 0,41 \\
\hline Prolapso & 2 & 3 & 1 & & 2,48 \\
\hline Sangrado & 3 & 7 & 1 & 1 & 4,97 \\
\hline Retención urinaria & 2 & 2 & & 1 & 2,07 \\
\hline Infección herida quirúrgica & 2 & 2 & & & 1,65 \\
\hline Extrusión de malla & 4 & 5 & 1 & 2 & 4,97 \\
\hline Síndrome vasovagal & 1 & & & & 0,41 \\
\hline Tetania & & 1 & & & 0,41 \\
\hline Paresia MMII & 1 & 1 & & & 0,82 \\
\hline
\end{tabular}

Global: TOT 21,27\%; TOT+CP 36,36\%; TOT+HV 33,3\%; TOT+HV+CP 17,02\%.

Acerca de la continencia y satisfacción de las mujeres a los 2 meses de la intervención, sólo disponemos de datos de 183 mujeres, que representan el $75,93 \%$. La tasa global de continencia es de un 72,13 $\%$ (132 mujeres) y de satisfacción de un 67,75\% (124 mujeres). A los seis meses, los datos disponibles son de 100 mujeres, lo que representa un 41,49\% de las mujeres intervenidas. En este tiempo, la tasa global de continencia es del 73\% (73 mujeres) y de satisfacción es del 70\% (70 mujeres) (Tabla 3). El grado de incontinencia fue evaluado en consulta de suelo pélvico por un urólogo experimentado, usando la encuesta validada en nuestro centro. Con respecto a la satisfacción se evaluó mediante entrevista clínica y en algunos casos de manera telefónica.

El análisis en función del tipo de IU se refleja en la Tabla 4.

\section{DISCUSIÓN}

En la literatura científica existen numerosos estudios $^{4-7}$ que avalan la igualdad de eficacia entre el procedimiento TVT y la vía transobturadora, de modo que este valor ronda el 85-90\% para ambos procedimientos, sin embargo la tasa de mejoría subjetiva de las pacientes es algo mayor en la TOT (79\%) respecto a la TVT $(73 \%)$. Además ambos procedimientos son similares en cuanto a la curva
Tabla 3. Continencia y satisfacción en función de la técnica empleada

\begin{tabular}{lcccc}
\hline & \multicolumn{2}{c}{$\mathbf{2}$ meses } & \multicolumn{2}{c}{$\mathbf{6}$ meses } \\
& $\begin{array}{c}\text { Continencia } \\
\%\end{array}$ & $\begin{array}{c}\text { Satisfacción } \\
\text { \% }\end{array}$ & $\begin{array}{c}\text { Continencia } \\
\%\end{array}$ & $\begin{array}{c}\text { Satisfacción } \\
\%\end{array}$ \\
\hline TOT & 76,8 & 72,46 & 72,2 & 69,69 \\
TOT+CP & 73,23 & 67,60 & 82,35 & 79,41 \\
TOT+HV & 50 & 50 & 60 & 40 \\
TOT+HV+CP & 67,74 & 64,51 & 64,28 & 64,28 \\
\hline
\end{tabular}


Tabla 4.Continencia en función de la técnica empleada y el tipo de IU previa a la cirugía.

\begin{tabular}{|c|c|c|c|c|c|c|}
\hline & \multicolumn{3}{|c|}{2 meses } & \multicolumn{3}{|c|}{6 meses } \\
\hline & \multicolumn{3}{|c|}{ Continentes } & \multicolumn{3}{|c|}{ Continentes } \\
\hline & $\begin{array}{c}\text { IUE } \\
\%\end{array}$ & $\begin{array}{c}\text { IUM } \\
\%\end{array}$ & $\begin{array}{c}\text { No IU } \\
\%\end{array}$ & $\begin{array}{c}\text { IUE } \\
\%\end{array}$ & $\begin{array}{c}\text { IUM } \\
\%\end{array}$ & $\begin{array}{c}\text { No IU } \\
\%\end{array}$ \\
\hline TOT & 76 & 86,6 & 50 & 76 & 62,5 & - \\
\hline $\mathrm{TOT}+\mathrm{CP}$ & 75,75 & 85,71 & 61,90 & 82,35 & 88,88 & 75 \\
\hline TOT+HV & 50 & 100 & 25 & 50 & - & 66 \\
\hline $\mathrm{TOT}+\mathrm{HV}+\mathrm{CP}$ & 85,71 & 20 & 20 & 75 & 50 & 62,5 \\
\hline Tasa global & 75,72 & 77,77 & 58,53 & 76,78 & 68 & 68,75 \\
\hline
\end{tabular}

nencia posterior de estas pacientes. Hay que destacar la baja tasa de éxito en pacientes con diagnostico de no incontinencia en el estudio urodinámico, probablemente el procedimiento quirúrgico les empeore al función y mucho más si se acompaña de otros procedimientos reparativos del suelo pélvico.

A la vista de nuestros resultados, la TOT podría ser útil incluso en aquellas mujeres carentes de un diagnóstico urodinámico de IU. En éstas, la tasa global de continencia oscila entre el $50-61,90 \%$ a los dos meses, llegando hasta el 75\% a los seis meses, en aquellos casos en los que se corrige algún defecto del suelo pélvico, ya sea con colporrafia o malla intravaginal.

En este estudio se confirma que la TOT es una técnica segura, con una tasa de perforación vesical del 2,48\%, y aunque en algún trabajo como el de Zullo MA et $\mathrm{al}^{8}{ }^{8}$, no tienen ninguna $(0 \%)$, también hay que tener en cuenta que su casuística es de tan sólo 37 casos. Además es una técnica bien tolerada por las pacientes, presentando 12 extrusiones de malla $(4,97 \%)$ que se resolvieron sin complicaciones. En los diversos estudios analizados es cierto que la tasa de complicaciones postoperatorias ronda el 5-10\%, mientras que nuestra tasa de complicaciones postoperatoria es del $26,55 \%$, esto sólo es justificado por la metodología del estudio, ya que mientras en otros trabajos solo consideran complicación mayores como las extrusiones, en nuestro estudio contabilizamos complicaciones mayores y menores, incluyendo, infecciones de orina, prolapsos, retención urinaria y dolores posquirúrgicos leves-moderados. A pesar de todo esto se ha constado mediante multitud de estudios clínicos e histológicos que el polipropileno es un material sintético bien tolerado por el cuerpo que no predispone a padecer infecciones o a erosionar los tejidos (en nuestro caso, vagina y uretra) ${ }^{9}$.

La tasa global de satisfacción a los dos y seis meses $(67,75 \%$ y $70 \%)$ es similar a la descrita por Roumeguèr T et al. ${ }^{10}(78 \%)$ y suele ser pareja a la tasa de continencia. Así podemos observar como la tasa más alta se obtiene cuando se realizamos la técnica aislada, rondando el 70\%; esta tasa desciende al aplicar otros procedimientos siendo más llamativa esta caída al realizar histerectomía vaginal junto con la TOT, lo que hace pensar que es la histerectomía lo que provoca cierta insatisfacción en las pacientes y no la TOT por sí misma.

Parece sensato pensar que a pesar de los buenos resultados obtenidos y la mejora evidente respecto a la TVT, son necesarios seguimientos a más largo plazo, quizá de esta misma muestra de pacientes, para establecer con mayor grado de evidencia y fiabilidad tanto la eficacia como la seguridad de éste tipo de procedimientos.

\section{REFERENCIAS}

1. Ulmsten U, Petros P. Intravaginal slingplasty (IVS): an ambulatory surgical procedure for treatment of female urinary incontinence. Scand J Urol Nephrol. 1995;29(1):75-82.

2. Delorme E. Transobturator urethral suspension: miniinvasive procedure in the treatment of stress urinary incontinence in women. Prog Urol. 2001;11(6):13061313.

3. Galmés Belmonte I, Díaz Gómez E. ¿Son iguales todos los sistemas empleados para corregir la incontinencia urinaria mediante mallas libres de tensión?. Actas Urol Esp. 2004;28(7):487-496.

4. Laurikainen E, Valpas A, Kivelä A, Kalliola T, Rinne K, Takala T, et al. Retropubic compared with transobturator tape placement in treatment of urinary incontinence: a randomized controlled trial. Obstet Gynecol. 2007;109(1):4-11.

5. Liapis A, Bakas P, Giner M, Creatsas G. Tension-free vaginal tape versus tensionfree vaginal tape obturator in women with stress urinary incontinence. Gynecol Obstet Invest. 2006;62(3): 160-164

6. Schanz Pardo J, Ricci Arriola P, Solà Dalenz V, Tacla Fernández X. Trans-obturator-tape (TOT) for the surgical repair of stress urinary incontinence: our experience. Arch Esp Urol. 2006;59 (3):225-232.

7. de Leval J, Waltregny D. New surgical technique for treatment of stress urinary incontinence TVT-Obturator: new developments and results. Surg Technol Int. 2005; 14:212-221.

8. Zullo MA, Plotti F, Calcagno M, Marullo E, Palaia I, Bellati F, et al. One-year followup of tension-free vaginal tape (TVT) and trans-obturator suburethral tape from inside to outside (TVT-O) for surgical treatment of female stress urinary incontinence: a prospective randomised trial. Eur Urol. 2007;51(5):1376-1382.

9. Delorme E, Droupy S, de Tayrac R, Delmas V. Transobturator tape (Uratape): a new minimally-invasive procedure to treat female urinary incontinence. Eur Urol. 2004;45(2):203-207.

10. Roumeguère T, Quackels T, Bollens R, de Groote A, Zlotta A, Bossche MV, et al. Trans-obturator vaginal tape (TOT) for female stress incontinence: one year follow-up in 120 patients. Eur Urol. 2005;48(5):805-809.

Correspondencia: Dr. I. Zapardiel Gutiérrez Departamento de Obstetricia y Ginecología. Hospital Universitario Santa Cristina

Maestro Vives, 2 - 28009 Madrid. Tel.: 0034667020440

E-mail autor: ignaciozapardiel@hotmail.com

Trabajo recibido: diciembre 2007

Trabajo aceptado: enero 2008 